\title{
Functional replacement of Trypanosoma brucei Argonaute by the human slicer Argonaute2
}

\author{
HUAFANG SHI, ${ }^{1}$ CHRISTIAN TSCHUDI, ${ }^{1,2}$ and ELISABETTA ULLU ${ }^{1,3}$ \\ Departments of ${ }^{1}$ Internal Medicine, ${ }^{2}$ Epidemiology \& Public Health and ${ }^{3}$ Cell Biology, \\ Yale University Medical School, New Haven, Connecticut 06536-0812, USA
}

\begin{abstract}
RNA interference (RNAi) is widespread throughout the eukaryotic lineage, from protozoa to man. Central to all RNAi phenomena is a member of the Argonaute protein family, and, in the case of dsRNA-triggered mRNA cleavage, the Ago protein functions as the RNAi endonuclease or slicer. However, at present there is no definite experimental evidence that slicer Argonautes can be interchanged between distantly related organisms. Here, we show that the human slicer Argonaute2 (HsAgo2), but not HsAgo1, functions in RNAi in the early divergent protozoan Trypanosoma brucei, thus mimicking the situation in mammalian cells. This finding indicates that the basic features of the RNAi mechanism are conserved from $T$. brucei to man.
\end{abstract}

Keywords: RNA interference; Argonaute; trypanosome; human Ago2; siRNAs

\section{INTRODUCTION}

In recent years RNA silencing mechanisms mediated by small 21- to 30-nt RNAs have emerged as crucial regulators of gene expression in eukaryotes. Transcription, heterochromatin formation, mRNA stability, and translation are among the processes that can be regulated by this class of small RNAs (Tomari and Zamore 2005 and references therein). At present the pathway of mRNA cleavage triggered by dsRNA, or RNA interference (RNAi) (Fire et al. 1998), is the best understood mode of RNA silencing at the mechanistic level. In RNAi, cleavage of the target transcript is executed by an effector complex termed "RNA-induced silencing complex," or "RISC" (Hammond et al. 2000). At the core of RISC is a member of the Argonaute (Ago) protein family complexed with the guide strand of a small interfering RNA (siRNA) or a micro RNA (miRNA) (for review, see Hall 2005), whose key feature is perfect complementarity to the target transcript (Hutvagner and Zamore 2002). Whereas it has been known for several years that the binding of the small RNA to its target specifies the site of cleavage (Elbashir et al. 2001), the endonucleolytic

Reprint requests to: Elisabetta Ullu, Department of Internal Medicine, Yale University Medical School, 295 Congress Avenue, New Haven, CT 06536-0812, USA; e-mail: elisabetta.ullu@yale.edu; fax: (203) 785-7329.

Article published online ahead of print. Article and publication date are at http://www.rnajournal.org/cgi/doi/10.1261/rna.20806. activity of RISC turned out to be more difficult to identify. However, recent biochemical experiments have clearly demonstrated that the human Ago2 protein is the RISC endonuclease or slicer (Liu et al. 2004; Meister et al. 2004; Rivas et al. 2005), with the active site residing within a predicted RNAse H-type fold of the Piwi domain (Song et al. 2004). More recently, the Drosophila Ago2 (Miyoshi et al. 2005) and Arabidopsis thaliana Agol (Baumberger and Baulcombe 2005) proteins have also been shown to be endowed with slicer activity. Finally, mutational analysis of the Agol protein in the protozoan parasite Trypanosoma brucei is consistent with its proposed activity as slicer (Shi et al. 2004c).

So far slicer activity is the only function attributed to a subset of eukaryotic Ago family members, and this enzymatic activity is clearly dependent on key residues in the Piwi domain of the various Ago proteins. However, outside the Piwi domain and the second characteristic domain of Ago proteins, namely PAZ (Cerutti et al. 2000), there is limited primary sequence conservation when slicer Agos from metazoan organisms are compared to the proposed slicer Ago protein of T. brucei (Shi et al. 2004c), a protozoon that is considered an early divergent eukaryote. Is this sequence divergence a reflection of functional differences between the aforementioned Ago proteins? To answer this question we tested whether human Ago2 (HsAgo2) could functionally replace T. brucei Ago1. We 
found that HsAgo2, but not HsAgo1, restored degradation of target mRNA in Agol-null trypanosomes, thus arguing that the basic features of the RNAi mechanism have been conserved throughout eukaryotic evolution.

\section{RESULTS AND DISCUSSION}

The T. brucei genome contains a single Ago protein (TbAgo1), which conforms to the domain structure of Ago family members and is essential for the RNAi response (Durand-Dubief and Bastin 2003; Shi et al. 2004b). Genetic ablation of TbAgol results in viable cells, although the loss of the RNAi pathway is accompanied by mild (Shi et al. 2004a) to severe (Durand-Dubief and Bastin 2003) growth defects, depending on the strain used for the experiments. At the functional level TbAgo-null trypanosomes lost the ability to mount an RNAi response, and this is accompanied by a prominent reduction of endogenous retroposon-derived 24- to 26-nt siRNAs and up-regulation of retroposon transcript levels (Shi et al. 2004b). However, a very small amount of endogenous siRNAs, which are a few nucleotides longer than the average size, is still detectable in $a g o 1^{-1-}$ cells. At present, the relationship between these different size classes of siRNAs is not understood. Lastly, mutational analysis of TbAgol showed that the R735 residue is essential for mRNA degradation in RNAi (Shi et al. 2004c). Since this residue is adjacent to one of the active site residues in the RNAse $\mathrm{H}$ fold (Song et al. 2003; Liu et al. 2004), we proposed that TbAgol functions as the trypanosome slicer.

Recently, the function of four human Ago family members, HsAgo 1-4, has been the object of several investigations. In particular, HsAgo2 is endowed with slicer activity, whereas no cleavage activity could be documented for the other three members of the family, although all four Ago proteins form complexes with miRNAs or siRNAs (Liu et al. 2004; Meister et al. 2004). In order to test whether human Ago2 could complement the RNAi deficiency of ago $^{-/-}$trypanosomes, we cloned HA-FLAG epitopetagged human Ago2 cDNA (Meister et al. 2004) into a trypanosome expression vector and generated stable clonal cell lines by integration at the tubulin locus of $a g o 1^{-1-}$ cells. As a control for these experiments we chose HsAgol and similarly produced clonal cell lines. Expression of the human proteins was verified by Western blot analysis using antiHA antibodies (Fig. 1A). We tested two independent clonal lines for each of the two transgenes and selected clones HsAgo1-a and HsAgo2-a (herein referred to as HsAgol and HsAgo2 cells) for further analysis, as these cell lines appeared to express the corresponding Ago proteins to similar levels. Next, we asked whether expression of the human Ago proteins restored accumulation of endogenous retroposon-derived siRNAs. As shown in Figure 1B, Northern blot analysis of RNA isolated from HsAgol (lane 3) or HsAgo2 (lane 4) cells showed accumulation of siRNA-like
A

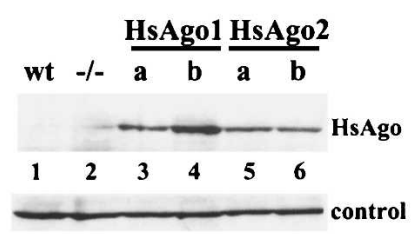

B

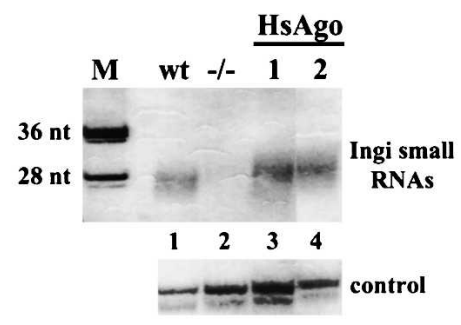

C

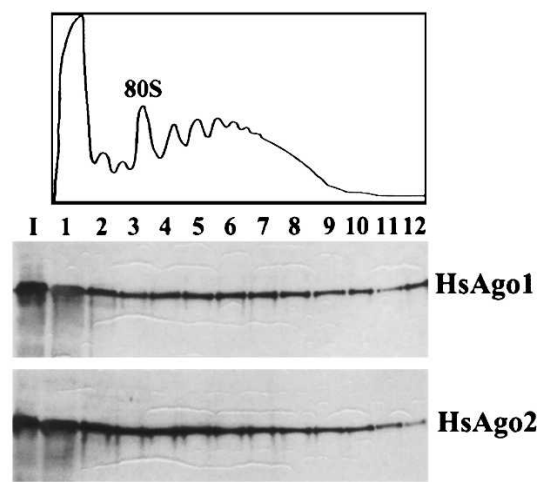

FIGURE 1. Expression of human Ago1 and Ago2 in Ago1-null trypanosomes. (A) Western blot analysis of whole-cell lysates from clonal lines of transgenic trypanosome expressing HsAgol (HsAgola and HsAgo1-b, lanes 3,4) or HsAgo2 (HsAgo2-a and HsAgo2-b, lanes 5,6). The human Ago proteins (HsAgo) were detected with antiHA antibodies. wt (lane 1), extract from T. brucei wild-type procyclic cells; -I- (lane 2), extract from ago1 ${ }^{-/-}$procyclic cells; control (bottom panel), a cross-reacting protein band that served as a loading control. (B) Expression of human Agol or Ago2 restores accumulation of retroposon-derived siRNA-like molecules in Agol-null trypanosomes. Low-molecular-weight RNA from wild-type (lane 1), ago1 $^{-1-}$ (lane 2), HsAgol (lane 3), or HsAgo2 (lane 4) cells was prepared as described previously (Shi et al. 2004b) and electrophoresed on a $15 \%$ sequencing gel. After transfer, the filter was hybridized with a radiolabeled sense riboprobe representing a portion of the Ingi retroelement. (M) 36- and 28-nt $3^{\prime}$-end labeled DNA fragments of pBR322 DNA digested with MspI. The panel labeled "control" shows the cross-hybridization to a background band, which served as a control for RNA loading. (C) Sucrose density gradients of cytoplasmic extracts from cells expressing HsAgol or HsAgo2. The upper panel shows the absorbance profile at $254 \mathrm{~nm}$ of one of the two gradients, and the position of the $80 \mathrm{~S}$ particle is indicated. The two panels below the absorbance profile show a Western blot analysis of the sucrose density gradient fractions using anti-HA antibodies.

molecules homologous to the endogenous retroposon Ingi. Curiously, in both instances the average size of these small RNAs was a few nucleotides longer than that of the siRNAs derived from wild-type cells (lane 1) and was similar to the average size of the residual siRNAs detected in $a g o 1^{-/-}$cells (barely visible in Fig. 1B, lane 2, but see also Shi et al. 
2004b). After correcting for the loading of RNA in each lane, the amount of Ingi siRNA-like molecules in HsAgol and HsAgo2 cells was comparable to that of wild-type trypanosomes. Furthermore, most of the siRNA-like molecules were in a complex with the corresponding human Ago protein as shown by immunoprecipitation of cell lysates with anti-FLAG antibodies (data not shown).

Since in trypanosomes a proportion of the Agol ribonucleoprotein complex is found associated with polyribosomes (Djikeng et al. 2003; Shi et al. 2004b), we next tested the sedimentation characteristics of HsAgol and HsAgo2 by centrifugation of cytoplasmic extracts on sucrose density gradients (Fig. 1C). Similar to what we observed for TbAgol, a proportion of both human Ago proteins sedimented as large ribonucleoprotein particles with a distribution superimposable onto that of polyribosomes. Whether the human Ago proteins interact with translating ribosomes will require further investigation. Nevertheless, it is interesting that these heterologous proteins have sedimentation features highly reminiscent of the endogenous TbAgol.

From the results shown in Figure 1B it appeared that expression of human Ago1 and Ago2 in T. brucei ago1 ${ }^{-1-}$ cells not only restored accumulation of Ingi siRNA-like molecules but also somehow influenced the size of these RNAs. There are several possible explanations for the latter observation. For instance, it is possible that the longer siRNA molecules are more stable than the shorter ones, thus resulting in a skewed representation of size classes in steady-state RNA. However, the finding that both in $a g o 1^{-/}$ trypanosomes (Shi et al. 2004b) and in HsAgo1 or HsAgo2 cells the size of siRNAs is longer than wild-type suggests the possibility that Agol in T. brucei is somehow involved in the biogenesis of siRNAs. Perhaps in $T$. brucei the size of siRNAs is influenced by an interaction between Agol and Dicer, either directly or through the intervention of a yet to be discovered dsRNA-binding protein analogous to the human tar-binding protein (TRBP) (Haase et al. 2005) or to Drosophila R2D2 (Liu et al. 2003). In support of this hypothesis biochemical studies indicate that human Ago2 and Dicer directly interact via the Piwi domain of Ago and the RNAse IIIa domain of Dicer (Tahbaz et al. 2004), although the precise function of this interaction is not understood. We recently identified T. brucei Dicer and found that this protein is considerably different in structure from the typical Dicer enzyme (H. Shi, C. Tschudi, and E. Ullu, in prep.). One possible explanation of our findings is that the human Ago proteins cannot engage in a fully productive interaction with the "atypical" T. brucei Dicer, giving rise to aberrantly sized siRNAs.

Notwithstanding the longer size of siRNAs, we next tested whether a functional RNAi response had been restored in HsAgol and HsAgo2 cells. As a first assay we transfected HsAgol and HsAgo2 cells with dsRNA corresponding to the $5^{\prime}$ UTR of $\alpha$-tubulin mRNA (Ngo et al. 1998) and, $16 \mathrm{~h}$ later, scored the cultures for the presence of the so-called FAT phenotype (cells with multiple nuclei, flagella, and mitochondrial genomes, which arise because of the transient down-regulation of $\alpha$-tubulin). As expected, wild-type cells were very sensitive to dsRNA, with $\sim 50 \%$ of the cells becoming FAT following exposure to $1 \mu \mathrm{g}$ of $\alpha$-tubulin dsRNA and reaching a maximum of $\sim 98 \%$ FAT cells at $5 \mu \mathrm{g}$ of dsRNA (Fig. $2 \mathrm{~A}$ ). In contrast, $a g o 1^{-/-}$cells, as reported previously (Shi et al. 2004b), did not respond to dsRNA; i.e., no FAT cells were observed, even when using an excess of $20 \mu \mathrm{g}$ of dsRNA. Similarly, challenging cells expressing HsAgol with different amounts of $\alpha$-tubulin dsRNA did not lead to the appearance of FAT cells.
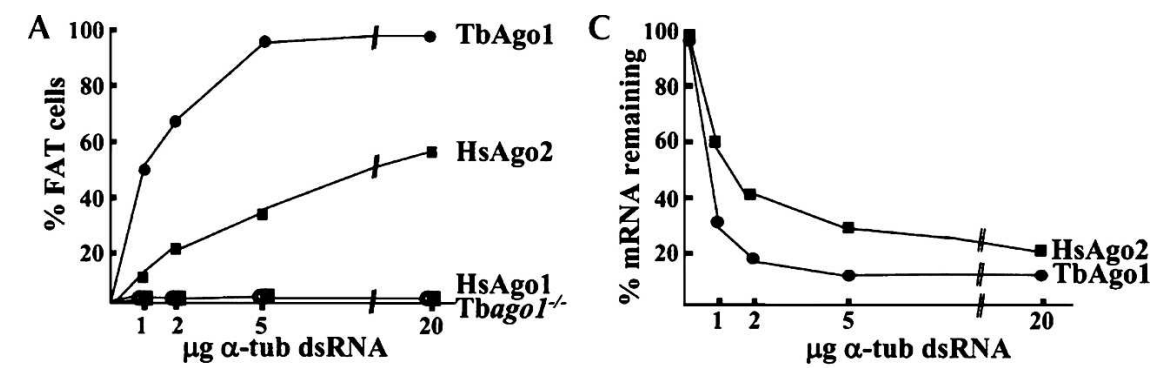

B

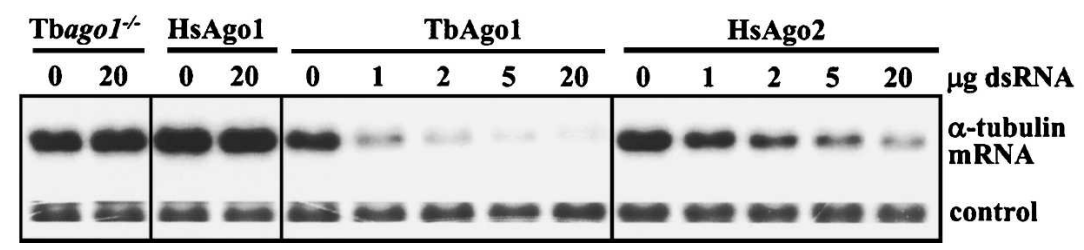

FIGURE 2. Expression of HsAgo2, but not of HsAgo1, restores RNAi competency in Ago1null trypanosomes. (A) Generation of FAT cells upon electroporation of $\alpha$-tubulin dsRNA. Wild-type (solid circles), Tbagol ${ }^{-l-}$ (open circles), HsAgo1-a (open squares), or HsAgo2a (solid squares) cells were electroporated with the indicated amounts of $\alpha$-tubulin dsRNA. After $16 \mathrm{~h}$ of growth, the percentage of FAT cells (cells with multiple nuclei and flagella) was determined by observation at the optical microscope and is plotted on the $Y$-axis. $(B)$ Disappearance of $\alpha$-tubulin mRNA triggered by $\alpha$-tubulin dsRNA. Wild-type (TbAgo1), Tbago $^{-1-}$, HsAgo1, or HsAgo2 cells were electroporated with different amounts (in micrograms) of $\alpha$-tubulin dsRNA as indicated above each lane or with poly $(I-C)$ (lane 0$)$, and total RNA was prepared $2 \mathrm{~h}$ after electroporation. The level of $\alpha$-tubulin mRNA was monitored by Northern blot with a radiolabeled DNA probe derived from the tubulin coding region. The bottom panel (control) shows the methylene blue staining of one of the large ribosomal RNAs that served as a loading control. (C) Quantitation of $\alpha$-tubulin mRNA disappearance in wildtype and HsAgo 2 cells. For each cell line $\alpha$-tubulin mRNA hybridization was quantitated by PhosphorImager analysis and was plotted as the fraction of mRNA remaining $2 \mathrm{~h}$ after electroporation, taking as $100 \%$ the amount of $\alpha$-tubulin mRNA present in the samples that received poly $(I-C)$ or $0 \mu \mathrm{g}$ of specific dsRNA. 
However, when we transfected cells expressing HsAgo2 with dsRNA, we noticed a significant number of FAT cells. In particular, at all dsRNA concentrations tested, HsAgo2 cells gave rise to FAT cells, reaching a maximum of $\sim 50 \%$ FAT cells at the highest dsRNA concentration tested.

To corroborate the FAT cell results, we next directly examined whether expression of HsAgo2 restored the ability of Ago-null trypanosomes to degrade target mRNA upon transfection of dsRNA. For this experiment, the various cell lines were electroporated with different amounts of $\alpha$-tubulin dsRNA, the fate of the targeted mRNA was monitored by Northern blot analysis (Fig. 2B), and the results were quantitated by PhosphorImager analysis (Fig. 2C). Consistent with the observed FAT cell phenotype, tubulin mRNA was efficiently targeted in wild-type cells but not in $a g o 1^{-/-}$cells or in cells expressing HsAgol. However, when HsAgo 2 was expressed in ago1 ${ }^{-/-}$cells, the mRNA degradation response was partially restored. Almost $75 \%$ of the tubulin mRNA was degraded when $20 \mu \mathrm{g}$ of tubulin dsRNA was transfected. Taken together, the results presented in Figure 2 showed that the human slicer HsAgo2, but not HsAgo1, was capable of substituting for the function of TbAgo1. Interestingly, the failure of HsAgo1 to compensate for TbAgol function mimicked what has been observed previously, namely that HsAgol has no detectable slicer activity in in vitro cleavage assays. However, the restoration of mRNA degradation by HsAgo2 was only partial. At all dsRNA concentrations tested the RNAi "competency" of HsAgo2 cells was about two- to threefold lower than what was observed in wild-type cells. Perhaps only a proportion of HsAgo 2 was functional in T. brucei, maybe because only the HsAgo 2 molecules bound to the correct size siRNAs were competent for cleavage. Needless to say, the lower than wild-type activity of HsAgo2 could be due to defects in other steps of the RNAi pathway, including recognition of the target mRNA and/or interaction with other, yet to be identified components of the T. brucei RNAi pathway. Nevertheless, our results imply that the basic features of the RNAi mechanism have been conserved between trypanosomes and man, organisms that are evolutionarily only distantly related.

\section{MATERIALS AND METHODS}

\section{Expression of HsAgo1 and HsAgo2 in T. brucei procyclic ago1 ${ }^{-/-}$cells}

The coding regions of HA-FLAG tagged human Ago1 and Ago2 cDNA were excised by digestion with EcoRI and HindIII from the corresponding plasmids pIRESneo-FLAG/HA-Ago1 or -Ago2 (Meister et al. 2004) and cloned into the trypanosome expression vector pXS2 ${ }^{\text {neo }}$ (Bangs et al. 1996). The resulting constructs were linearized with MluI for integration at the tubulin locus and transfected into ago1 $^{-/-}$cells (Shi et al. 2004b). Stable clonal cell lines for each of the two transgenes were derived by limited dilution.
Expression of the human Ago proteins was verified by Western blot analysis of whole-cell lysates using anti-FLAG or anti-HA antibodies. Sucrose density gradient centrifugation of cytoplasmic extracts was carried out as described (Shi et al. 2004b).

\section{RNA analysis}

Northern blot analyses and transfection of tubulin dsRNA were carried out as previously described (Shi et al. 2004b).

\section{ACKNOWLEDGMENTS}

We thank Dr. Thomas Tuschl, who kindly provided the HsAgol and HsAgo 2 cDNA clones. This work was supported by NIH grant AI28798 to E.U.

Received January 16, 2006; accepted February 8, 2006.

\section{REFERENCES}

Bangs, J.D., Brouch, E.M., Ransom, D.M., and Roggy, J.L. 1996. A soluble secretory reporter system in Trypanosoma brucei. Studies on endoplasmic reticulum targeting. J. Biol. Chem. 271: 18387-18393.

Baumberger, N. and Baulcombe, D.C. 2005. Arabidopsis ARGONAUTE1 is an RNA Slicer that selectively recruits microRNAs and short interfering RNAs. Proc. Natl. Acad. Sci. 102: 11928-11933.

Cerutti, L., Mian, N., and Bateman, A. 2000. Domains in gene silencing and cell differentiation proteins: The novel PAZ domain and redefinition of the Piwi domain. Trends Biochem. Sci. 25: 481-482.

Djikeng, A., Shi, H., Tschudi, C., Shen, S., and Ullu, E. 2003. An siRNA ribonucleoprotein is found associated with polyribosomes in Trypanosoma brucei. RNA 9: 802-808.

Durand-Dubief, M. and Bastin, P. 2003. TbAGO1, an Argonaute protein required for RNA interference, is involved in mitosis and chromosome segregation in Trypanosoma brucei. BMC Biol. 1: 2.

Elbashir, S.M., Lendeckel, W., and Tuschl, T. 2001. RNA interference is mediated by $21-$ and 22-nucleotide RNAs. Genes \& Dev. 15: 188-200.

Fire, A., Xu, S., Montgomery, M.K., Kostas, S.A., Driver, S.E., and Mello, C.C. 1998. Potent and specific genetic interference by doublestranded RNA in Caenorhabditis elegans. Nature 391: 806-811.

Haase, A.D., Jaskiewicz, L., Zhang, H., Laine, S., Sack, R., Gatignol, A., and Filipowicz, W. 2005. TRBP, a regulator of cellular PKR and HIV-1 virus expression, interacts with Dicer and functions in RNA silencing. EMBO Rep. 6: 961-967.

Hall, T.M. 2005. Structure and function of argonaute proteins. Structure 13: 1403-1408.

Hammond, S.M., Bernstein, E., Beach, D., and Hannon, G.J. 2000. An RNA-directed nuclease mediates post-transcriptional gene silencing in Drosophila cells. Nature 404: 293-296.

Hutvagner, G. and Zamore, P.D. 2002. A microRNA in a multipleturnover RNAi enzyme complex. Science 297: 2056-2060.

Liu, Q., Rand, T.A., Kalidas, S., Du, F., Kim, H.E., Smith, D.P., and Wang, X. 2003. R2D2, a bridge between the initiation and effector steps of the Drosophila RNAi pathway. Science 301: 1921-1925.

Liu, J., Carmell, M.A., Rivas, F.V., Marsden, C.G., Thomson, J.M., Song, J.J., Hammond, S.M., Joshua-Tor, L., and Hannon, G.J. 2004. Argonaute2 is the catalytic engine of mammalian RNAi. Science 305: 1437-1441.

Meister, G., Landthaler, M., Patkaniowska, A., Dorsett, Y., Teng, G., and Tuschl, T. 2004. Human Argonaute2 mediates RNA cleavage targeted by miRNAs and siRNAs. Mol. Cell 15: 185-197.

Miyoshi, K., Tsukumo, H., Nagami, T., Siomi, H., and Siomi, M.C. 2005. Slicer function of Drosophila Argonautes and its involvement in RISC formation. Genes \& Dev. 19: 2837-2848. 
Ngo, H., Tschudi, C., Gull, K., and Ullu, E. 1998. Double-stranded RNA induces mRNA degradation in Trypanosoma brucei. Proc. Natl. Acad. Sci. 95: 14687-14692.

Rivas, F.V., Tolia, N.H., Song, J.J., Aragon, J.P., Liu, J., Hannon, G.J., and Joshua-Tor, L. 2005. Purified Argonaute2 and an siRNA form recombinant human RISC. Nat. Struct. Mol. Biol. 12: 340-349.

Shi, H., Chamond, N., Tschudi, C., and Ullu, E. 2004a. Selection and characterization of RNA interference-deficient trypanosomes impaired in target mRNA degradation. Eukaryot. Cell 3: 14451453.

Shi, H., Djikeng, A., Tschudi, C., and Ullu, E. 2004b. Argonaute protein in the early divergent eukaryote Trypanosoma brucei: Control of small interfering RNA accumulation and retroposon transcript abundance. Mol. Cell. Biol. 24: 420-427.

Shi, H., Ullu, E., and Tschudi, C. 2004c. Function of the trypanosome Argonaute 1 protein in RNA interference requires the N-terminal
RGG domain and arginine 735 in the Piwi domain. J. Biol. Chem. 279: 49889-49893.

Song, J.J., Liu, J., Tolia, N.H., Schneiderman, J., Smith, S.K., Martienssen, R.A., Hannon, G.J., and Joshua-Tor, L. 2003. The crystal structure of the Argonaute2 PAZ domain reveals an RNA binding motif in RNAi effector complexes. Nat. Struct. Biol. 10: 1026-1032.

Song, J.J., Smith, S.K., Hannon, G.J., and Joshua-Tor, L. 2004. Crystal structure of Argonaute and its implications for RISC slicer activity. Science 305: 1434-1437.

Tahbaz, N., Kolb, F.A., Zhang, H., Jaronczyk, K., Filipowicz, W., and Hobman, T.C. 2004. Characterization of the interactions between mammalian PAZ PIWI domain proteins and Dicer. EMBO Rep. 5: 189-194.

Tomari, Y. and Zamore, P.D. 2005. Perspective: Machines for RNAi. Genes \& Dev. 19: 517-529. 

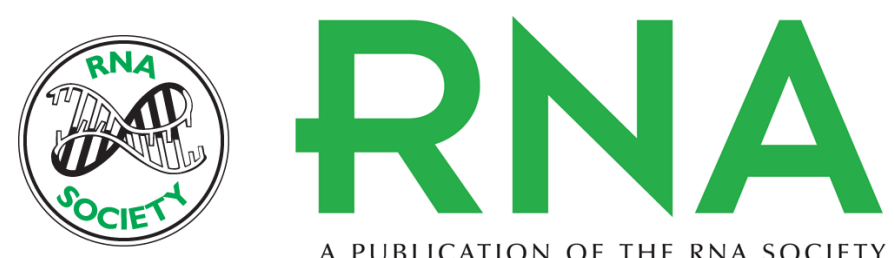

A PUBLICATION OF THE RNA SOCIETY

\section{Functional replacement of Trypanosoma brucei Argonaute by the human slicer Argonaute2}

Huafang Shi, Christian Tschudi and Elisabetta Ullu

RNA 2006 12: 943-947

References This article cites 24 articles, 14 of which can be accessed free at:

http://rnajournal.cshlp.org/content/12/6/943.full.html\#ref-list-1

License

Email Alerting Receive free email alerts when new articles cite this article - sign up in the box at the Service top right corner of the article or click here.

To subscribe to RNA go to:

http://rnajournal.cshlp.org/subscriptions 